\title{
A Re-investigation to the Death Line of Radio Pulsars
}

\author{
G. J. Qiao, Y. Q. Xue, B. Zhang, R. X. Xu, F. F. Ye, and H. G. Wang \\ Department of Astronomy, Peking University, Beijing 100871, \\ P.R.China.
}

\begin{abstract}
A new death line for radio pulsars is presented in this paper within the framework of vacuum gap and inverse Compton scattering (ICS) induced pair production process. The $8.5 \mathrm{~s}$ period pulsar PSR J2144-3933 is located above the death line without any additional assumptions. An "appearance line" instead of the so-called "Hubble line", is also presented in this paper. Both of those two lines fit observations well.
\end{abstract}

\section{Introduction}

Recently a long period $(P=8.51 s)$ radio pulsar PSR J2144-3933 has been observed (Young et al., 1999). For a given magnetic configuration, one can define a so-called "death line" in the $P-\dot{P}$ diagram ( $\dot{P}$, the pulsar spin down rate). Above the line one can observe radio pulsars in this region, which is called "radioloud" region. All previously known pulsars are located at the radio-loud region. Under the line no one can observe radio pulsars at all (radio-quiet). Since PSR J2144-3933 lies at the place where it seems to be out of the radio-loud region, the authors of the discovery pointed out that under the usual model assumptions, its pulsed emission could not be observed at radio wavelength. Therefore either the model assumptions were wrong, or current theories of radio emission should be revised. After the discovery, Zhang et al. (1999) re-investigated the radio pulsar death line within the framework of two different types of polar cap acceleration models. They found that the long period pulsar could be radio loud under ICS induced space-charge-limited flow model. Gil \& Mitra (2001) argued that the formation of vacuum gaps require a complicated surface magnetic field. Here we present a new death line under the framework of vacuum inner gap model and ICS induced pair production under the dipole magnetic configuration.

\section{Potential drop and acceleration in the inner gap}

Any theoretical model of radio pulsars should have a mechanism to accelerate the particles to a relativistic velocity. The equations in the inner gap model used here are as follows.

Potential drop in the inner gap. In the inner vacuum gap model, there is a strong electric field parallel to the magnetic field lines. The particles produced through $\gamma-B$ process in the gap can be accelerated and get very high energy ( e.g. $\gamma \sim 10^{6}$ ). The potential across the gap is $V=\frac{\Omega B}{c} h^{2}$, where $\Omega$ is 
the angular frequency of the neutron star; $h$ the gap height; $B, c$ represent the magnetic field at the surface of the neutron star and light speed respectively. As $h$ increases and approaches $r_{p}$, the potential drop along a field line traversing the gap can reach a maximum value (Ruderman \& Surtherland 1975, here after RS75)

$$
\triangle V_{\max }=\frac{\Omega B}{2 c} r_{p}^{2}
$$

where $r_{p}$ is the radius of the polar cap.

Acceleration in the inner gap. With acceleration by the electric field, particles produced in the gap can be accelerated and the Lorentz factor can be very high, namely $\gamma=\frac{e \triangle V}{m_{e} c^{2}}$, where $m_{e}$ is the mass of an electron or positron, $e$ the electric charge of an electron.

\section{The death line of radio pulsars}

In the inner gap model, $\gamma$-rays process plays a very important role. Three conditions should be satisfied at the same time for pair productions: (a) to produce high energy $\gamma$-rays, a strong enough potential drop should be reached; (b) the component of $\gamma$-rays perpendicular to the magnetic field must satisfy $E_{\gamma, \perp} \geq 2 m_{e} c^{2} ;$ (c) for pair production, usually the $\gamma-B$ process dominates other processes (Zhang \& Qiao 1998). With these criterions the death line will be re-investigated below.

Pair production in dipole magnetic field configuration and the death line. In $\gamma-B$ process, the mean photon free path in strong magnetic field is given by (Erber 1966):

$$
\begin{gathered}
l=\frac{4.4}{e^{2} / \hbar c} \frac{\hbar}{m_{e} c} \frac{B_{q}}{B_{\perp}} \exp \left(\frac{4}{3 \chi}\right),(\chi \ll 1) \\
\chi \equiv \frac{E_{\gamma}}{2 m_{e} c^{2}} \sin \theta \frac{B}{B_{q}}=\frac{E_{\gamma}}{2 m_{e} c^{2}} \frac{B_{\perp}}{B_{q}} .
\end{gathered}
$$

Here $B_{q}=4.414 \times 10^{13}$ Guass is the critical magnetic field, $\hbar$ the Planck's constant. $B_{\perp}$ is the magnetic field perpendicular to the moving direction of $\gamma$ photons, which can be expressed as $B_{\perp} \approx \frac{h}{\rho} B \approx \frac{l}{\rho} B$ (RS75). Here $h$ is the height of the gap, $l \approx h$ is assumed as the condition for the sparks to be produced, $\rho$ is curvature radius of the magnetic field lines. For dipole magnetic configuration, it is $\rho \approx \frac{4}{3}(\lambda R c / \Omega)^{1 / 2}$ (Zhang et al. 1997). Here, $\lambda$ is a parameter to define the field lines, $\lambda=1$ corresponds to the "last opening field" line. In the ICS process, the energy of gamma-rays is $E \gamma \approx 2 \gamma^{2} \hbar \omega\left(1-\beta \cos \theta_{i}\right)$, where $\beta=\frac{v}{c}, \gamma=\frac{1}{\left(1-\beta^{2}\right)^{1 / 2}}, v$ is the speed of particles, $\theta_{i}$ the incident angle of the incoming photons. For dipole magnetic field, the magnetic field can be written as $B=3.2 \times 10^{19}(P \dot{P})^{1 / 2}$ Guass. From the equations above we have

$$
A P^{3.5} \dot{P}^{-1.5}=D+\frac{1}{2} \ln \dot{P}-\ln P,
$$




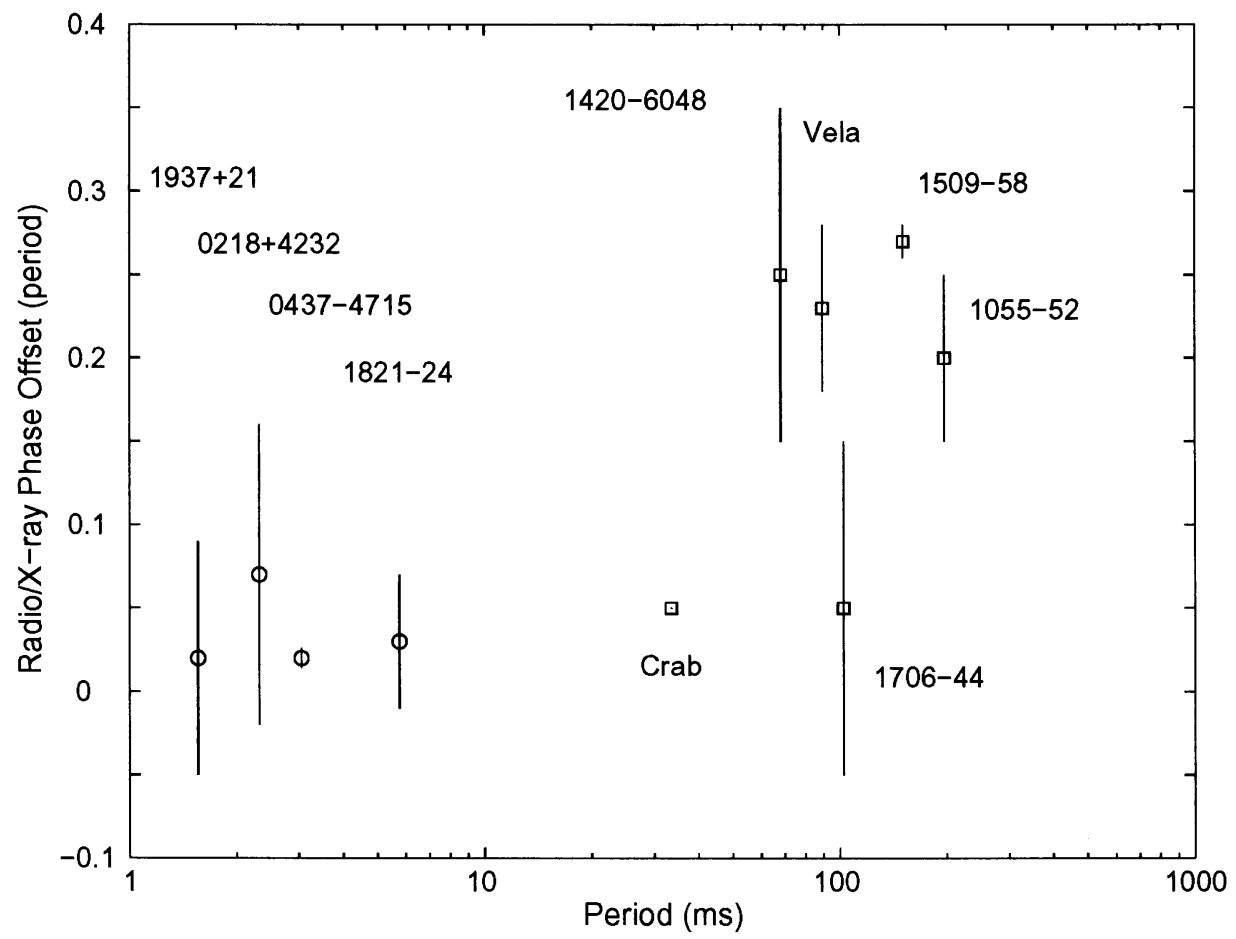

Figure 1. The "death line" and the "appearance line" on the $P-\dot{P}$ diagram for over 1300 pulsars.

where $A, D$ are constants. Here $r=\lambda r_{c} \sin ^{2} \theta$. If we take the field line at $0.8 \theta_{p}$ and $l=h=a r_{p}=0.5 r_{p}$, we get

$$
1.2 \times 10^{-25} P^{3.5} \dot{P}^{-1.5}=60.5+\ln \dot{P}-2 \ln P .
$$

With the Eq.(5), one can get a so-called death line in Fig.1. Above the line, neutron stars should be radio-loud; below the line, neutron stars should be radioquiet. The death line in Fig.1 can keep all observed pulsars, including the 8.5s pulsar, in the radio-loud region. If we adopt $l=h=0.8 r_{p}$, the corresponding line will be parallel to and a little lower than the death line in Fig.1. Both of them fit observations well.

Pair production through $\gamma-B$ process: multi-pole magnetic field. For the situation that multi-pole magnetic fields exist near the surface of the neutron stars, the curvature radius of the magnetic field is $\rho \approx 10^{6} \mathrm{~cm}$, we have $\frac{8}{3} \frac{1}{G} P^{3} \dot{P}^{-15}=2 \ln F+\ln \dot{P}-\ln P$, where $F, G$ are constants. With $B=3.2 \times$ $10^{19}(P \dot{P})^{1 / 2}$ Gauss ("dipole" magnetic configuration) and $l=0.3 r_{p}$, one can get $1.3 \times 10^{-26} P^{3} \dot{P}^{-1.5}=68.0+\ln \dot{P}-\ln P$. If we take $B=6.4 \times 10^{19}(P \dot{P})^{1 / 2}$ Gauss (viz. not "dipole", Shapiro et al. 1983 and Usov et al. 1995) and $l=0.3 r_{p}$, we get $1.6 \times 10^{-27} P^{3} \dot{P}^{-1.5}=69.4+\ln \dot{P}-\ln P$. The two lines are much lower than the death line in the Fig.1. For $l=0.5 r_{p}$ and $l=0.8 r_{p}$, the lines are further lower. 


\section{The "appearance line" of radio pulsars}

In the $\gamma-B$ process, the magnetic energy density should be high enough. We assume that the perpendicular magnetic energy density is not less than the pair energy density, that is $B_{\perp}^{2} / 8 \pi \geq 2 m_{e} c^{2} n_{p h}$, where $B_{\perp}$ is the component of the magnetic field perpendicular to the $\gamma$-rays, $n_{p h}$ is the number density of photons, that produce pairs in the $\gamma-B$ process. When the temperature is taken as $T=10^{5} k$, the photon number density is $n_{p h}=\frac{2.4}{\pi^{2}}\left(\frac{k T}{\hbar c}\right)^{3}$. We adopt dipole magnetic configuration and have (for $l=0.5 r_{p}$ )

$$
\log \dot{P}-\log P=-18.7
$$

The line corresponding to Eq.(6) is shown in Fig.1. When $l=0.8 r_{p}$, the line will be lower than that.

\section{Conclusion and discussion}

It is shown in this paper that the $8.5 \mathrm{~s}$ radio pulsar can be located at the radioloud region under the inner vacuum gap model without any additional assumptions. In our analysis, dipole magnetic configuration is used, multi-polar magnetic component can more easily make all observed pulsars locate in the radioloud region, but it is not necessary. An "appearance line" is also presented in this paper, which has a clear physical meaning: above the line pair production process can take place, so pulsars there are radio-loud; under the line the pair production process can not take place, so neutron stars there are radio-quiet. Near the place of the "appearance line" a so-called "Hubble Line" can often be found in the literature, but we doubt its physical meaning because the pulsars above the line are considered as "recycled pulsars", both pulsar period and period derivative are changed in the accretion.

Acknowledgments. This work is partly supported by NSF of China, the Climbing project, the National Key Basic Research Science Foundation of China and the Research Fund for the Doctoral Program Higher Education.

\section{References}

Erber, T. 1966, Rev.of.Mod.Phys., 38, 4

Gil, J., Mitra, D. 2001, ApJ, 550, 383

Shapiro, S.L., Teukolsky, S.A. 1983, JBAA, 93R, 276S

Usov, V.V.; Melrose, D.B. 1995, AuJPh, 48, 571U

Young, M.D., Manchester, R.N., Johnsoton, S. 1999, Nature, 400, 848

Zhang, B., Harding, A.K., Muslimov, A.G. 1999, AAS, 19513303Z

Zhang, B., Qiao, G.J., Han, J.L. 1997, ApJ, 491, 891

Zhang, B., Qiao, G.J., Han, J.L. 1998, Stas.conf, 385Z 